\title{
Comunicación
}

\section{Seroprevalencia de Mycoplasma agalactiae y Toxoplasma gondii en caprinos y ovinos de los municipios del sur de La Guajira y norte del Cesar, Colombia}

\author{
Prevalence of Mycoplasma agalactiae and Toxoplasma gondii in goats and \\ sheep in La Guajira and Cesar, Colombia
Omar Domínguez-Amorochoo, Wilson Diaz ${ }^{1,5}$ Julio Cesar Tobón², Diego Ortíz María Cristina Vásquez ${ }^{1}$

\section{Resumen}

\begin{abstract}
El estudio tuvo como objetivo determinar la prevalencia de Toxoplasma gondii y Mycoplasma agalactiae en ovinos y caprinos del sur de La Guajira y norte del Cesar como parte del establecimiento de un perfil serológico de estas especies animales en la región colombiana. Las muestras de suero $(n=1039)$ fueron analizadas con kits comerciales de ELISA específicos para estos dos patógenos. La prevalencia global encontrada en la región fue del 25.1 y $18.3 \%$ para $T$. gondii y de 0.12 y $0.8 \%$ para $M$. agalactiae, en ovinos y caprinos, respectivamente. Muestras positivas a $T$. gondii fueron más frecuentes en hembras que en machos. Asimismo, todas las muestras positivas a M. agalactiae fueron determinadas en hembras.
\end{abstract}

Palabras clave: Mycoplasma agalactiae, Toxoplasma gondii, caprinos, ovinos, plan sanitario, toxoplasmosis

\footnotetext{
${ }^{1}$ Universidad de Santander UDES, Programa de Bacteriología y laboratorio Clínico, Bucaramanga, Santander, Colombia

${ }^{2}$ Empresa Colombiana de Productos Veterinarios - VECOL, Bogotá, Colombia

${ }^{3}$ Universidad de Santander UDES, Programa de Medicina Veterinaria, Bucaramanga, Santander, Colombia

${ }^{4}$ Corporación Colombiana de Investigación Agropecuaria, CORPOICA, Bogotá, Colombia

${ }^{5}$ E-mail: o.dominguez@mail.udes.edu
}

Recibido: 14 de diciembre de 2020

Aceptado para publicación: 10 de julio de 2021

Publicado: 24 de agosto de 2021

CLos autores. Este artículo es publicado por la Rev Inv Vet Perú de la Facultad de Medicina Veterinaria, Universidad Nacional Mayor de San Marcos. Este es un artículo de acceso abierto, distribuido bajo los términos de la licencia Creative Commons Atribución 4.0 Internacional (CC BY 4.0) [https:// creativecommons.org/licenses/by/4.0/deed.es] que permite el uso, distribución y reproducción en cualquier medio, siempre que la obra original sea debidamente citada de su fuente original 
The aim of this study was to determine the prevalence of Toxoplasma gondii and Mycoplasma agalactiae in sheep and goats from the south of La Guajira and north of Cesar as part of the establishment of a serological profile of these species in the Colombian region. Serum samples $(\mathrm{n}=1039)$ were analysed with commercial ELISA kits specific for these pathogens. The global prevalence found in the region was 25.1 and $18.3 \%$ for $T$. gondii and 0.12 and $0.8 \%$ for M. agalactiae in sheep and goats, respectively. Positive samples for $T$. gondii were more frequent in females than in males. Likewise, all samples positive for M. agalactiae were determined in females.

Key words: Mycoplasma agalactiae, Toxoplasma gondii, health calendar, sheep, toxoplasmosis

\section{INTRODUCCIÓN}

Los departamentos de la Guajira y Cesar son considerados los principales productores de ovinos y caprinos en Colombia, pues según censo del Instituto Colombiano Agropecuario (ICA) para el 2015 se encontraban registrados 1108937 cabras y 1318241 ovejas, de las cuales el departamento de la Guajira cuenta con 886475 cabras y 615529 ovejas, mientras que el departamento del Cesar registra 20257 cabras y 71283 ovejas (ICA, 2015). Si bien los caprinos y ovinos no lideran el campo de la producción agropecuaria, son animales de interés sanitario no solo por los productos obtenidos de ellos, sino también por la capacidad que tienen como reservorio de enfermedades, algunas de curso zoonótico.

En este estudio, agentes infecciosos como Toxoplasma gondii y Mycoplasma agalactie revisten importancia, particularmente dado por la resolución 3714 de 2015 «Por la cual se establecen las enfermedades de declaración obligatoria en Colombia», estos agentes se consideran microorganismos de interés sanitario, no solo desde el punto de vista de sanidad animal, sino también, desde la perspectiva de la salud pública (ICA, 2015). En el caso de $T$. gondii, en ovinos y caprinos es común que se manifieste causando abortos o nacimiento de animales débiles (AbuDalbouh et al., 2012), afectando a los ganaderos, además de ser un problema de salud pública (Aguirre et al., 2019; Dubey et al., 2020). También deben ser consideradas las afectaciones de tipo congénita, así como la afectación en individuos inmunocomprometidos (Baranowski et al., 2014; Wang et al., 2017). Por su parte, Mycoplasma agalactiae causa una patología llamada agalaxia contagiosa, siendo el principal responsable de la mastitis en ovejas y cabras, lo que conlleva a una baja producción láctea. Además, puede producir artritis, queratoconjuntivitis, septicemia y muerte, e incluso aborto (Jaÿ y Tardy, 2019).

Diversos estudios han demostrado que estas enfermedades generan altos costos en términos de tratamiento, así como pérdidas por mortalidad y descarte, generando un impacto importante en la economía de los productores y comercializadores de los animales y sus subproductos (Dubey et al., 2020; Mee at al., 2021). Ante esto y basado a la escasa información en el país sobre las enfermedades infecciosas en ovinos y caprinos, se requiere determinar la seroprevalencia de la toxoplasmosis y la agalaxia contagiosa mediante el perfil serológico en los departamentos de Guajira y Cesar, con el fin de apor- 
tar información para el diseño de un plan sanitario direccionado hacia la prevención y el control de estas enfermedades, siguiendo los lineamientos de las autoridades sanitarias nacionales y el cumplimiento de las normativas internacionales (Caroprese et al., 2010; Sinclair, 2017).

\section{Materiales y Métodos}

\section{Área de Estudio y Animales}

El estudio se llevó a cabo en los municipios al sur de los departamentos de La Guajira y norte del Cesar, donde su actividad económica depende de la ganadería ovina y caprina.

El presente trabajo corresponde a un estudio descriptivo de corte transversal. Para el cálculo del tamaño muestral se tomó la población definida por el censo de 2015, correspondiente a 39000 ovinos y caprinos del sur del departamento de La Guajira y norte del departamento del Cesar (ICA, 2015). El tamaño de muestra se calculó con el Programa WinEpi v. 2.0 (Atlanta, USA), teniendo en cuenta una prevalencia esperada del $50 \%$, un error esperado del $3 \%$ y un nivel de confianza del $95 \%$, dando como resultado un tamaño de muestra de 1039 individuos.

\section{Muestras}

El muestreo fue realizado durante el segundo semestre de 2016. Las muestras de sangre fueron colectadas de la vena yugular por un médico veterinario utilizando tubos secos con sistema Vacutainer ${ }^{\circledR}$, evitando la hemolisis. Las muestras fueron transportadas al laboratorio de medicina veterinaria de la Universidad de Santander, sede Valledupar, donde el suero fue separado por centrifugación en un periodo inferior a 2 horas desde la toma de la muestra. Los sueros fueron almacenados a $-20{ }^{\circ} \mathrm{C}$ hasta el momento del envío al laboratorio donde serían procesados.
Las muestras fueron colocadas en una cava de material resistente con geles refrigerantes según NTC 4702-6 (ICONTEC, 1999) y enviadas al Laboratorio de Investigaciones Biomédicas y Biotecnológicas (LIBB) de la Universidad de Santander, sede Bucaramanga, en donde se mantuvieron a $-20{ }^{\circ} \mathrm{C}$, para su posterior procesamiento. La cadena de frio fue mantenida durante todo el proceso de separación, envío y procesamiento. Las muestras fueron transportadas por grupos dependiendo de las fechas de tomas de muestra y por regiones de muestreo.

Las muestras fueron analizadas mediante la técnica de ELISA para la determinación de IgG específica mediante el uso de los kits PRIOCHECK ${ }^{\circledR}$ Toxoplasma Ab SR (PRIONICS, Suiza) e IDEXX M. agalactiae verification (IDEXX, Francia), siguiendo el protocolo definido por los fabricantes. La lectura de los resultados se obtuvo por medio de espectrofotometría con lector de microplacas (BIO-RAD) a una longitud de onda de 450 $\mathrm{nm}$. El análisis de la información del ELSA se hizo mediante el programa estadístico IBM SPSS (Madrid, España).

\section{Aspectos Éticos}

Los caprinos y ovinos que participaron en este estudio fueron tratados de acuerdo con la normativa del Estatuto Nacional de Protección Animal en Colombia, Ley 84 de 1989 , por la cual se adopta y se regula la integridad de los animales (Congreso de Colombia, 1989) y bajo la aprobación y observación del Comité de Bioética de la Universidad de Santander UDES.

\section{Resultados}

El $76 \%$ de las 1039 muestras fueron tomadas a ovinos en tanto que el $24 \%$ restante a caprinos. En el caso de ovinos, $92.4 \%$ correspondieron a hembras y $7.6 \%$ a machos, y en el caso de caprinos el $89.4 \%$ fue a hembras y $10.6 \%$ a machos 
Cuadro 1. Seroprevalencia de Toxoplasma gondii en ovinos y caprinos de los departamentos del Cesar y La Guajira, Colombia (2016)

\begin{tabular}{ccccc}
\hline \multirow{2}{*}{ Departamento } & \multirow{2}{*}{ Especie } & \multicolumn{2}{c}{ Muestras } & \multirow{2}{*}{ Seroprevalencia } \\
\cline { 3 - 4 } & & Colectadas (n) & Positivas (n) & \\
\hline \multirow{3}{*}{ Cesar } & Caprino & 180 & 40 & 22.2 \\
& Ovino & 551 & 129 & 23.4 \\
& Caprino & 66 & 5 & 7.6 \\
& Ovino & 242 & 70 & 29.0 \\
\hline
\end{tabular}

Cuadro 2. Seroprevalencia de Toxoplasma gondii en ovinos y caprinos de los departamentos del Cesar y La Guajira, Colombia, según el sexo (2016)

\begin{tabular}{ccccc}
\hline \multirow{2}{*}{ Especie } & \multirow{2}{*}{ Especie } & \multicolumn{2}{c}{ Muestras } & \multirow{2}{*}{ Seroprevalencia } \\
\cline { 3 - 4 } & & Colectadas (n) & Positivas (n) & \\
\hline \multirow{2}{*}{ Caprino } & Hembra & 220 & 42 & 19.1 \\
& Macho & 26 & 3 & 11.5 \\
\multirow{2}{*}{ Ovino } & Hembra & 733 & 189 & 25.8 \\
& Macho & 60 & 10 & 16.7 \\
\hline
\end{tabular}

Se encontraron 244 muestras positivas a $T$. gondii de las 1039 analizadas. La seroprevalencia en ovinos fue de $25.1 \%$ y en caprinos de $18.4 \%$. La seroprevalencia encontrada para ovinos del departamento del Cesar fue similar, pero en el caso del departamento de La Guajira, la seroprevalencia en ovinos fue mayor (29.0) que en caprinos (7.6\%) (Cuadro 1). La seroprevalencia en ovinos fue mayor en hembras $(25.8 \%)$ que en machos (16.7\%), siendo similar distribución para el caso de los caprinos (19.1 en hembras y $11.5 \%$ en machos) (Cuadro 2).

En el caso de para M. agalactiae solo se encontraron tres muestras positivas, resultando una seroprevalencia global de $0.28 \%$. Esto es $0.27 \%$ en Cesar y de $0.32 \%$ en Gua- jira (Cuadro 3). Todas las muestras que resultaron positivas correspondieron a animales hembras.

\section{Discusión}

La producción ovina y caprina es una fuente económica importante en la región del Cesar y La Guajira. Estas dos regiones conforman junto con el Magdalena el «triángulo», donde está ubicado el $70 \%$ de la población ovinocaprina de Colombia (Moreno y Grajales, 2017).

La mayor seroprevalencia de infección por $T$. gondii, en comparación con la infección por $M$. agalactiae, especialmente en 
Cuadro 3. Seroprevalencia de Mycoplasma agalactiae en ovinos y caprinos de los departamentos del Cesar y La Guajira, Colombia (2016)

\begin{tabular}{ccccc}
\hline \multirow{2}{*}{ Departamento } & \multirow{2}{*}{ Especie } & \multicolumn{2}{c}{ Muestras } & \multirow{2}{*}{ Seroprevalencia } \\
\cline { 3 - 4 } & & Colectadas (n) & Positivas (n) & \\
\hline \multirow{3}{*}{ Cesar } & Caprino & 180 & 2 & 1.1 \\
& Ovino & 551 & 0 & 0 \\
& Caprino & 66 & 0 & 0 \\
& Ovino & 242 & 1 & 0.4 \\
\hline
\end{tabular}

ovinos podría deberse a la forma de pastar del ovino, ya que consumen vegetación casi a ras del suelo, lugar donde con frecuencia se encuentran los ooquistes del parásito, a diferencia del caprino que tiende a consumir pasto o ramas, que crecen separados de la tierra, disminuyendo la probabilidad de contraer el agente etiológico por vía oral (Gebremedhin et al., 2014). En este sentido, Hamilton et al. (2014) en islas del Caribe encontraron una mayor frecuencia de casos positivos para $T$. gondii en ovinos (59\%) que en caprinos $(52.9 \%)$.

La mayor seroprevalencia de infección por $T$. gondii en hembras puede estar relacionada con los niveles bajos en la respuesta inmune en algunos periodos de su vida (Yin et al., 2015). Resultados similares fueron encontrados por van der Puije et al. (2000) en Ghana. Dado el potencial zoonótico que tiene el parásito, y las complicaciones que genera en las mujeres embarazadas y personas inmunocomprometidas, la seroprevalencia observada en las zonas en estudio pasa a convertirse en una situación de interés en términos de salud pública.

La seroprevalencia de $M$. agalactiae fue bastante baja, con solo tres casos positivos; no obstante, la seroprevalencia de este patógeno se reporta de manera muy variada en otras regiones. Así, Giangaspero et al. (2012) no encontraron casos positivos Japón, mientras que Al-Momani et al. (2008) en Jordania reportaron altas seroprevalencias en ovinos y caprinos, en tanto que Assunção et al. (2004) observaron una seroprevalencia de $55 \%$ en caprinos en las Islas Canarias.

Si bien la infección por $M$. agalactiae no representa un peligro para los humanos, implica un impacto desfavorable para el ganadero, ya que en la mayoría de los casos los animales deben ser sacrificados. Además, la infección por este microorganismo es de notificación obligatoria a la Organización Mundial de Sanidad Animal (OIE). Cabe resaltar que a la fecha no se ha realizado otros estudios que permitan actualizar o estimar la seroprevalencia de estos agentes en Colombia.

\section{Agradecimientos}

Los autores agradecen a la Universidad de Santander UDES y la Empresa Colombiana de Productos Veterinarios VECOL por el soporte financiero y logístico del estudio. Asimismo, agradecen a Oscar Porras, Leidy Chávez, Julieth Peñaranda y Andrea Roa de la Universidad de Santander UDES y a Alfredo Sánchez de VECOL por su contribución técnica durante el estudio. 


\section{Literatura Citada}

1. Abu-Dalbouh MA, Ababneh MM, Giadinis ND, Lafi SQ. 2012. Ovine and caprine toxoplasmosis (Toxoplasma gondii) in aborted animals in Jordanian goat and sheep flocks. Trop Anim Health Prod 44:49-54. doi: 10.1007/s11250-011-9885-2

2. Aguirre AA, Longcore T, Barbieri M, Dabritz, H, Hill D, Klein PN, Lepczyk C, et al. 2019. The One Health approach to toxoplasmosis: epidemiology, control, and prevention strategies. EcoHealth 16: 378-390. doi: 10.1007/ s10393-019-01405-7

3. Al-Momani W, Nicholas R.A, AboShehada M.N. 2008. Risk factors associated with Mycoplasma agalactiae infection of small ruminants in northern Jordan. Prev Vet Med 83: 110. doi: 10.1016/j.prevetmed.2007.08.00

4. Assunção P, De la Fe C, Ramirez AS, Andrada M, Poveda JB. 2004. Serological study of contagious agalactia in herds of goats in the Canary Islands. Vet Rec 154: 684-687. doi: 10.1136/ vr.154.22.684

5. Baranowski E, Bergonier D, Sagné E, Hygonenq MC, Ronsin P, et al. 2014 Experimental infections with Mycoplasma agalactiae identify key factors involved in host-colonization. Plos One 9(4): e93970. doi: 10.1371/journal.pone.0093970

6. Caroprese M, Donato C, Rassu S, Napolitano F, Sevi A. 2010. Monitoring the on-farm welfare of sheep and goats. It J Anim Sci 8. doi: 10.4081/ijas.2009.s1.343

7. Congreso de Colombia. 1989. Ley 84 de 1989. [Internet]. Disponible en: http:/ /www.alcaldiabogota.gov.co/sisjur/normas/Norma1.jsp?i=8242

8. Dubey JP, Murata FHA, CerqueiraCézar CK, Kwok OCH, Yang YR. 2020. Public health significance of-
Toxoplasma gondii infections in cattle: 2009-2020. J Parasitol 106: 772-788. soi: $10.1645 / 20-82$

9. Gebremedhin EZ, Abdurahaman M, Hadush T, Tessema TS. 2014. Seroprevalence and risk factors of Toxoplasma gondii infection in sheep and goats slaughtered for human consumption in Central Ethiopia. BMC Res Notes 7: 696. doi: 10.1186/1756-0500-7-696

10. Giangaspero M, Nicholas RA, Hlusek $M$, Bonfini B, Osawa T, Orusa $R$, Tatami S, et al. 2012. Seroepidemiological survey of sheep flocks from Northern Japan for Mycoplasma ovipneumoniae and Mycoplasma agalactiae. Trop Anim Health Pro 44: 395398. doi: 10.1007/s11250-011-9952-8

11. Hamilton CM, Katzer F, Innes EA, Kelly PJ. 2014. Seroprevalence of Toxoplasma gondii in small ruminants from four Caribbean islands. Parasite Vector 7: 449. doi: 10.1186/1756-33057-449

12. [ICA] Instituto Colombiano Agropecuario. 2015. Censo Nacional Pecuario. [Internet]. Disponible en: http:// www.ica.gov.co/getdoc/8232c0e5be9742bd-b07b-9cdbfb07fcac/Censos2008.aspx.

13. [ICA] Instituto Colombiano Agropecuario. 2015. Resolución 3714 de 2015. Por la cual se establecen las enfermedades de declaración obligatoria en Colombia. [Internet]. Disponible en: https://fenavi.org/documentos/ resolucion-3714-de-2015/.

14. [ICONTEC] Instituto Colombiano de Normas Técnicas y Certificación. 1999. Embalajes y envases para transporte de mercancías peligrosas Clase 6: sustancias tóxicas e infecciosas. [Internet]. Disponible en: https:// tienda.icontec.org/gp-embalajes-y-envases-para-transporte-de-mercancias-peligrosas-clase-6-sustancias-toxicas-e-infecciosas-ntc4702-6-1999.html 
15. Jä̈ M, Tardy F. 2019. Contagious agalactia in sheep and goats: current perspectives. Vet Med (Auckl) 10: 229247. doi.: 10.2147/VMRR.S201847

16. Mee JF, Jawor P, Stefaniak T. 2021. Role of infection and immunity in bovine perinatal mortality: part 1. Causes and current diagnostic approaches. Animals (Basel) 11: 1033. doi: 10.3390/ ani11041033

17. Moreno DC, Grajales HA. 2017. Caracterización de los sistemas de producción ovinos de trópico alto en Colombia: manejo e indicadores productivos y reproductivos. Rev Fac Med VetZootec 64:36-51. doi: 10.15446/ rfmvz.v64n3.-68693

18. Sinclair M. 2017. Internationalization of animal welfare standards. In: Thompson P, Kaplan D (eds). Encyclopedia of Food and Agricultural Ethics. Springer. doi: 10.1007/978-94-007-6167-4_572-2
19. van der Puije WN, Bosompem KM, Canacoo EA, Wastling JM, Akanmori BD. 2000. The prevalence of antiToxoplasma gondii antibodies in Ghanaian sheep and goats. Acta Trop 76: 21-26. doi: 10.1016/s0001-706x(00)00084-x

20. Wang ZD, Liu HH, Ma ZX, Ma HY, Li ZY, Yang ZB, Zhu XQ, et al. 2017. Toxoplasma gondii infection in immunocompromised patients: a systematic review and meta-analysis. Front Microbiol 8: 389. doi: 10.3389/ fmicb.2017.00389

21. Yin MY, Wang JL, Huang SY, Qin SY, Zhou DH, Liu GX, Tan QD, Zhu XQ. 2015. Seroprevalence and risk factors of Toxoplasma gondii in Tibetan sheep in Gansu province, Northwestern China. BMC Vet Res 11: 41. doi: 10.1186/ s12917-015-0358-0. 